\title{
KPiX - A 1,024 Channel Readout ASIC for the ILC
}

\author{
J. Brau, M. Breidenbach*, A. Dragone, G. Fields, R. Frey, D. Freytag, M. Freytag, C. Gallagher,
} G. Haller, R. Herbst, B. Holbrook, R. Lander, A. Moskaleva, C. Neher, T. Nelson, S. Schier, B. Schumm, D. Strom, M. Tripathi, M. Woods

\begin{abstract}
KPiX is a 1,024 channel "System on a Chip" intended for bump bonding to large area Si sensors, enabling low multiple scattering $\mathrm{Si}$ strip tracking and high density Particle Flow calorimetry for $\mathrm{SiD}$ at the International Linear Collider (ILC). It may be used for hadronic calorimetry readout with RPC's or GEM's, and with a scintillator-based muon system using SiPM's. An electromagnetic calorimeter prototype will be beam-tested in early 2013.
\end{abstract}

\section{INTRODUCTION}

$\mathrm{T}_{\mathrm{H}}$ Silicon Detector (SiD) [1], shown in quadrant view in Fig. 1, is intended for the International Linear Collider (ILC). SiD features a cost optimized design emphasizing high momentum resolution tracking and high spatial resolution jet calorimetry based on Particle Flow Algorithms to exploit the physics potential of an $\mathrm{e}^{+} \mathrm{e}^{-}$collider with energies up to $\sqrt{\mathrm{s}}_{\mathrm{s}}=1$ $\mathrm{TeV}$.

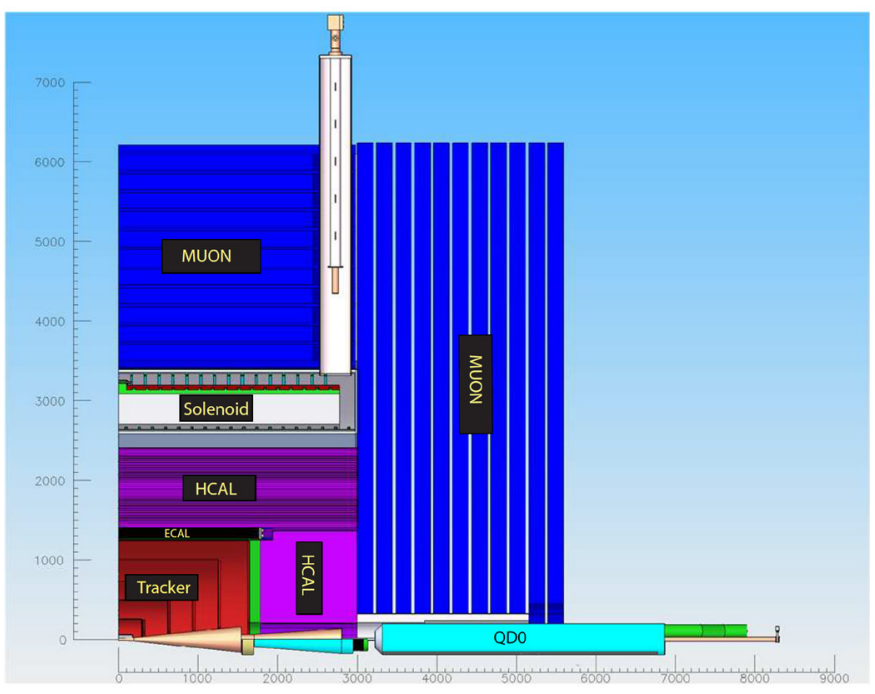

Fig. 1. Quadrant view of SiD.

The tracker consists of a 5-layer barrel and two 4-layer endcaps. The barrel sensors are $10 \mathrm{~cm}$ square Si strip sensors with 50 micron readout pitch, each with two KPiX chips and a thin power and data cable as shown in Fig. 2. The sensors are held in low mass clips, and are then mounted on carbon fiber support cylinders.

Manuscript received November 16, 2012. SLAC-PUB-15285.

M. Breidenbach*, A. Dragone, D. Freytag, M. Freytag, G. Haller, R. Herbst and T. Nelson are with the SLAC National Accelerator Laboratory, Menlo Park, CA 94025 USA (* corresponding author, telephone: 650-9262872, e-mail: mib@slac.stanford.edu). G. Fields, B. Holbrook, R. Lander, A. Moskaleva, C. Neher, M. Tripathi and M. Woods are with University of California, Davis. S. Schier and B. Schumm are with Santa Cruz Institute for Particle Physics and the University of California at Santa Cruz. J. Brau, R. Frey, C. Gallagher and D. Strom are with University of Oregon.

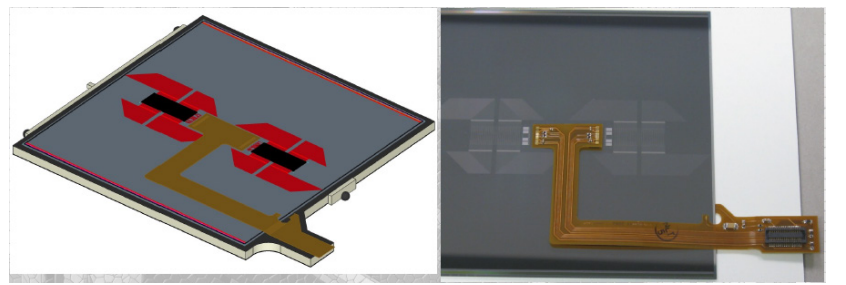

Fig. 2. Views of the $\mathrm{SiD}$ tracker sensor design with two KPiX and flex cable.

The electromagnetic calorimeter consists of $26 \mathrm{X}_{0}$ tungsten in 30 plates $(20 \times 2.5 \mathrm{~mm}$ followed by $10 \times 5 \mathrm{~mm})$, interleaved with Si detectors as shown in Fig. 3.

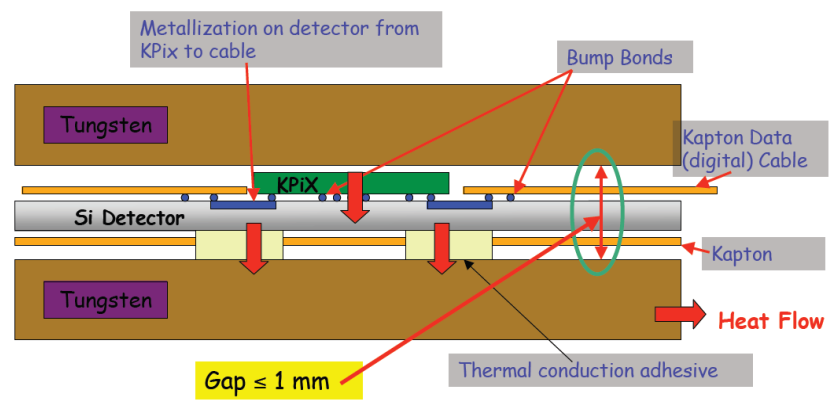

Fig. 3. Detail of $\mathrm{SiD}$ electromagnetic calorimeter (ECAL), showing tungsten plates interleaved with Si sensors.

The calorimeter gaps are expected to be less than $1.25 \mathrm{~mm}$, optimizing the Moliere radius and minimizing the radial extent of the calorimeter. The readout for the tracker and calorimeter, and possibly other detector systems of $\mathrm{SiD}$, is based on KPiX [2], a 1,024 channel 0.25 micron mixed-mode process CMOS ASIC "System on a Chip". The architecture of KPiX takes explicit advantage of the beam structure of the ILC: $\sim 3,000$ pulses during a $1 \mathrm{~ms}$ train, repeating at $5 \mathrm{~Hz}$. The analog front end current is modulated during the $199 \mathrm{~ms}$ intertrain period to a low power state, saving about a factor of 100 in average power consumption.

\section{THE KPIX ASIC}

A simplified block diagram of KPiX is shown in Fig. 4. Each channel consists of a dynamically switchable gain charge amplifier; shaping; threshold discrimination; and 4 sample and hold capacitors and 4 timing registers. The chip permits 4 separate measurements of amplitude and time of threshold crossing during each train, and amplitude digitization and readout during the intertrain period. The dynamic range is 


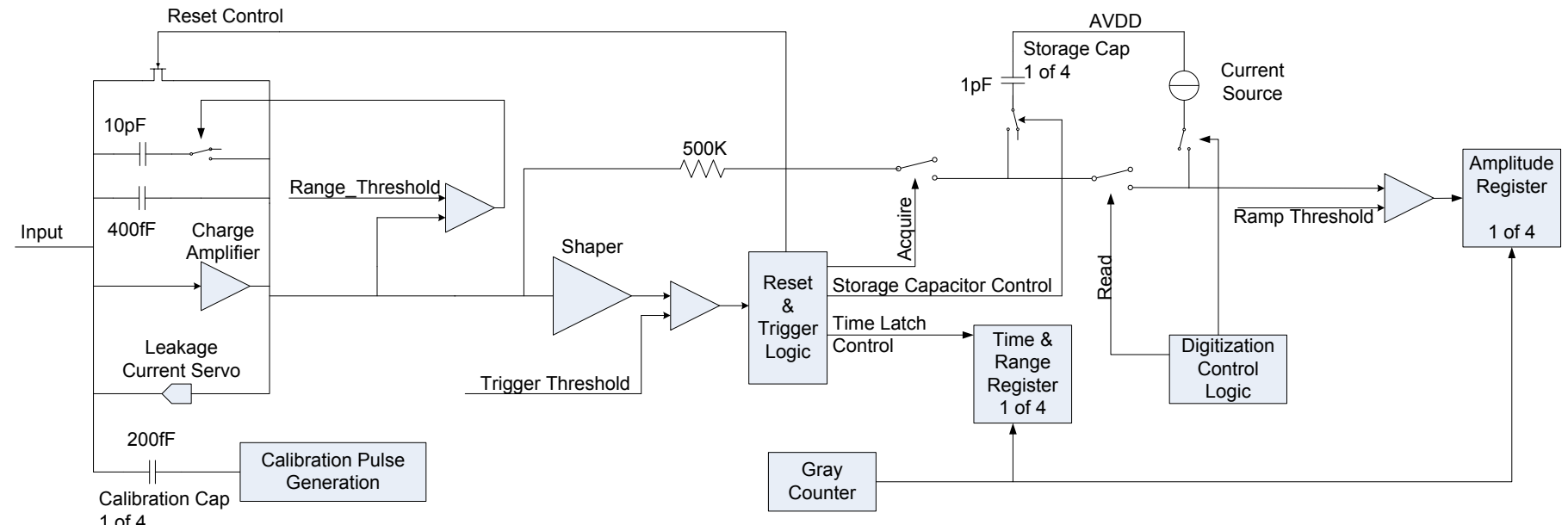

Fig. 4. Simplified block diagram of a single KPiX readout channel (out of 1024 channels).

from sub minimum ionizing particle (mip) (320 micron silicon) to more than 2,000 mip. In normal operation, the charge amplifier is synchronously reset after each bunch crossing. KPiX also has a calibration system for each channel, servos for leakage compensation, "DC" reset for asynchronous operation for testing with cosmic rays, and polarity inversion for use with GEMs and similar detectors. The noise floor is about $0.15 \mathrm{fC}(\sim 1,000$ electrons $)$, and the maximum signal is $10 \mathrm{pC}$ (utilizing the dynamic range switching). The full dynamic range corresponds to 17 bits.

More details of the circuit are discussed in [2].

$\mathrm{KPiX}$ is intended to be bump bonded to the various $\mathrm{Si}$ sensors. Sn-Pb eutectic bumps on a 200 x 500 micron lattice are placed in wells on each KPiX pad by the foundry [3]. All sensor signals are routed to corresponding pads on a second metal layer, and pads and traces are also provided for power, clock, control, external trigger, and data signals to connect to a bump bonded cable. Thus there is no hybrid, and the resulting assembly for the electromagnetic calorimeter sensor, shown in Fig. 6, is less than $1 \mathrm{~mm}$ thick.

KPiX is a possible readout system for the hadronic calorimeter, where it would be integrated with PC boards having $1 \mathrm{~cm}$ by $1 \mathrm{~cm}$ pads. It is also being considered for the $\mathrm{SiD}$ muon system, likely in a 64-channel version, reading scintillator strips with SiPM sensors. While these applications do not require the precise amplitude measurements of the tracker and electromagnetic calorimeter, there appears to be no cost penalty for the extra information.

The average power consumption of a KPiX in the ILC duty cycle is $<20 \mathrm{~mW}$. This permits the tracker to be gas cooled (total power $<600$ watts), and the electromagnetic calorimeter is cooled by conduction through the tungsten plates to water loops on the edges. This level of integration of the sensor and readout system essentially enables the low multiple scattering tracker and dense calorimeter of SiD.

Tracker and calorimeter prototype sensors were manufactured by Hamamatsu [4] with Al pads. Suitable Under Bump Metallization (UBM) proved extremely difficult; conventional zincate or sputtering processes failed to produce a usable gold surface. A cleaning and sputtering process by
IZM [5] appears to be satisfactory, and measurements using the first prototype are presented here. An x-ray image of the bonds is shown in Fig. 5.

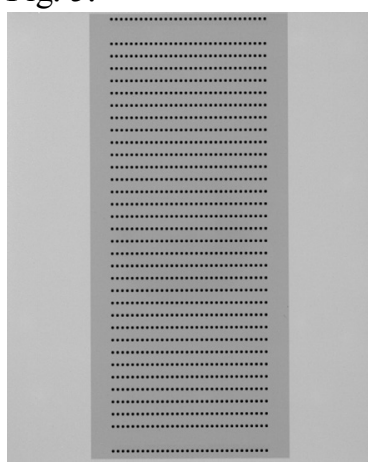

Fig. 5. X-ray image of the bump bond array between KPiX and an Electromagnetic Calorimeter sensor.

A cable assembly incorporating strain relief (for the thermal cycle of the attachment) was bump bonded to the sensor, as shown in Fig. 6.

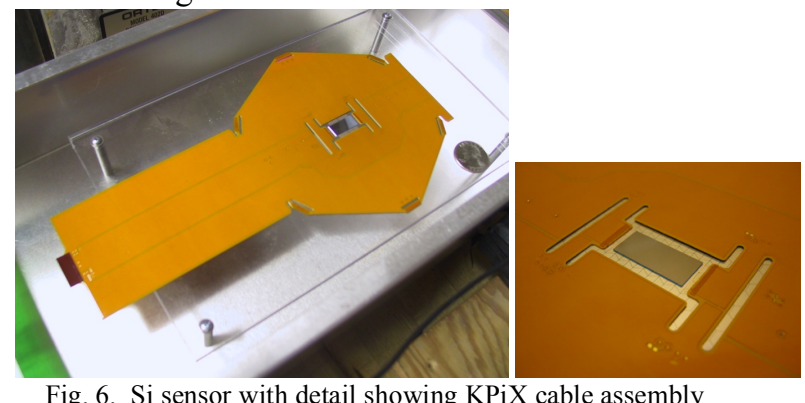

Fig. 6. Si sensor with detail showing KPiX cable assembly

Mechanical prototype sensors with some inoperative pixels have been used for these first assembly tests.

Fig. 7 shows first results obtained with a cosmic ray telescope. The KPiX is externally triggered by the telescope which is larger than the sensor. For $25 \%$ of the triggers, one of the pixels is traversed by a cosmic ray. The blue curve shows data with the telescope physically displaced from the sensor, showing the largest noise signal for each trigger from all the working pixels. The red curve shows data with the sensor aligned inside the telescope, and the curve is a Landau fit with 
a Most Probable Value of $3.7 \mathrm{fC}$, which is expected for a 320 micron Si sensor. Since $75 \%$ of the triggers do not have a hit pixel, there is still a large spike near zero measuring the noise. The blue curve is normalized to that spike.

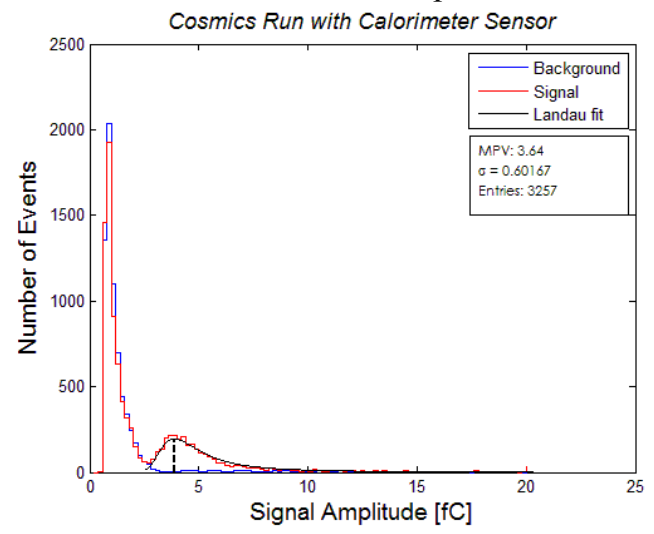

Fig. 7. Data taken by a cosmic ray telescope that uses KPiX for readout

Fig. 8a shows the residual noise distributions of an unconnected KPiX. The data agree with a Gaussian of sigma $=0.16 \mathrm{fC}$ over five orders of magnitude. Fig. $8 \mathrm{~b}$ shows residuals for KPiX bonded to a sensor. The Gaussian is wider (sigma $=0.23 \mathrm{fC}$ ) due to the capacitive load of the pixels. At the $1 \%$ rate-level tails develop, mostly due to some noisy pixels.
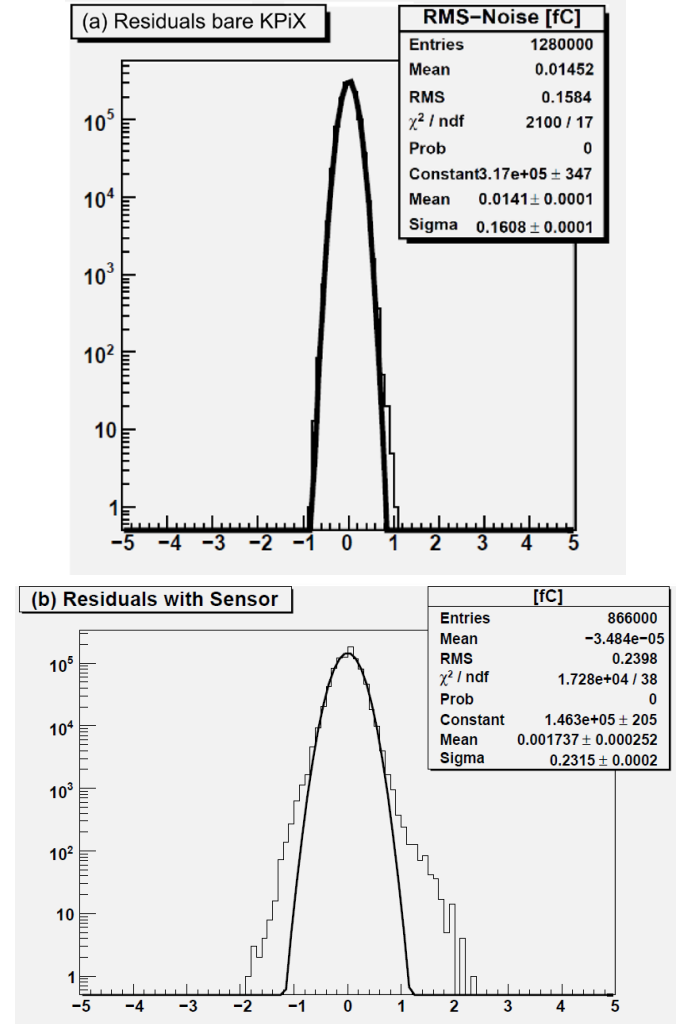

Fig. 8. Residual noise distributions for the bare KPiX and for KPiX bonded to a sensor.

The KPiX calibration system is used to measure crosstalk with KPiX bonded to the sensor. Four pixels receive a charge of $500 \mathrm{fC}$ and the response of all remaining pixels is measured. Fig. 9 shows in blue the residuals when the calibration charge is set to zero, together with a Gaussian fit to the data. The red curve shows all pixels except the four pulsed ones. The mean has shifted by $-0.04 \mathrm{fC}$ and a few more events show up in the tails four orders of magnitude down from the peak.

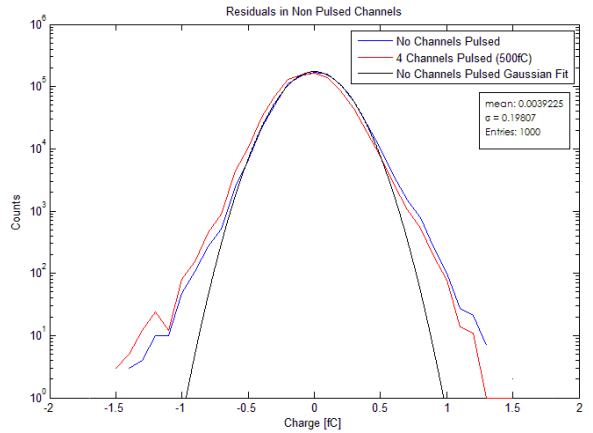

Fig. 9. Residuals of spectator pixels with (red) and without (blue) the four $500 \mathrm{fC}$ calibration signals, plus a Gaussian fit to the data.

Fig. 10 shows data with cosmic rays, but in self triggered mode. Events were accepted when the timing flag agreed with the telescope timing. This proved to be a powerful selection tool against background-noise triggers.

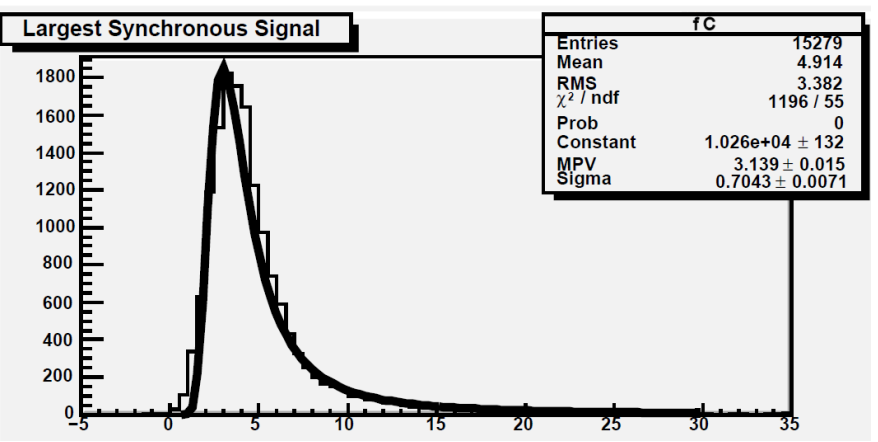

Fig. 10. Sensor response to cosmic rays in KPiX self-triggered mode.

The collaboration is now in the process of preparing 30 sensors for a test beam calorimeter, as shown in Fig. 11.

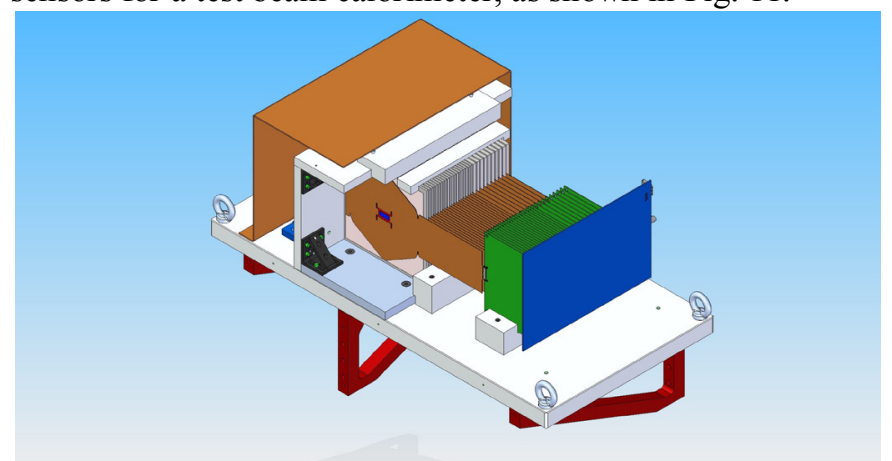

Fig. 11. Model of the Electromagnetic Calorimeter beamtest prototype.

This stack has the same depth profile as that expected for $\mathrm{SiD}$, but is only one sensor transverse. It is expected to be tested at the SLAC End Station Test Beam (ESTB), shown schematically in Fig. 12, in early 2013. The testbeam will provide clean, synchronous, high energy secondary electrons intended for sensor testing. 


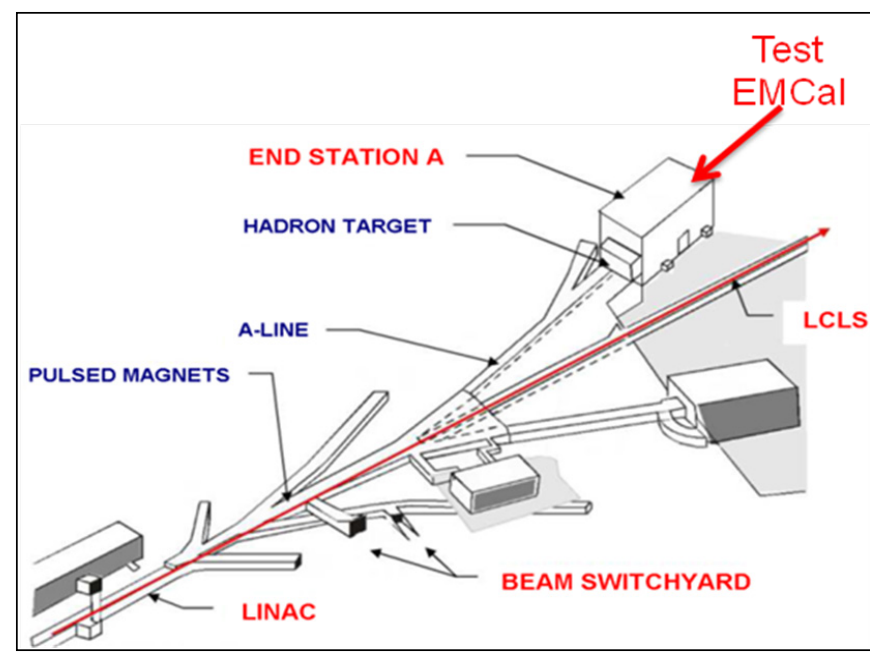

Fig. 12. SLAC End Station Test Beam (ESTB), showing location of test EM calorimeter at End Station A.

\section{ACKNOWLEDGMENT}

The authors would like to thank Guadalupe Salgado, Tung Phan and Mark Arndt from SLAC for their continuing support to the project.

Use of the SLAC National Accelerator Laboratory was supported by the U.S. Department of Energy, Office of Science, Office of Basic Energy Sciences, under Contract No. DE-AC02-76SF00515.

\section{REFERENCES}

[1] SiD Letter of Intent. H. Aihara, P. Burrows, M. Oreglia, editors. Internet: http://lcdev.kek.jp/IDAG/SiDLoI.pdf. May 4, 2012.

[2] KPiX, An Array of Self Triggered Charge Sensitive Cells Generating Digital Time and Amplitude Information. D. Freytag, et al.. Dec 11, 2008. 4 pp. SLAC-PUB-13462. Presented at Conference: C08-10-18

[3] Taiwan Semiconductor Manufacturing Company Limited. http://www.tsmc.com/english/default.htm.

[4] Hamamatsu Photonics. http://www.hamamatsu.com/. May 4, 2012.

[5] Fraunhofer Institute for Reliability and Microintegration. http://www.izm.fraunhofer.de/en.html. 